\title{
Hierarchical Photonic Pigments via the Confined Self-Assembly of Bottlebrush Block Copolymers
}

Dong-Po Song*,†, Tianheng H. Zhao ${ }^{\dagger}$, Giulia Guidetti, Silvia Vignolini* and Richard M. Parker

$\dagger$ These authors contributed equally.

Dr. D.-P. Song*

Tianjin Key Laboratory of Composite and Functional Materials, School of Materials Science and Engineering, Tianjin University, Tianjin 300350, People's Republic of China.

* E-mail: dpsong33@gmail.com

T. H. Zhao, Dr. G. Guidetti, Dr. S. Vignolini,* Dr. R. M. Parker

Department of Chemistry, University of Cambridge, Lensfield Road, Cambridge, CB2 1EW, UK.

*Email:sv319@cam.ac.uk 


\begin{abstract}
Hierarchical, structurally colored materials offer a wide variety of visual effects that cannot be achieved with standard pigments or dyes. However, their fabrication requires simultaneous control over multiple length-scales. Here we introduce a robust strategy for the fabrication of hierarchical photonic pigments via the confined self-assembly of bottlebrush block copolymers within emulsified microdroplets. The bottlebrush block copolymer self-assembles into highly ordered concentric lamellae, giving rise to a near perfect photonic multi-layer in the solid-state, with reflectivity up to $100 \%$. The reflected color can be readily tuned across the whole visible spectrum by either altering the molecular weight or by blending the bottlebrush block copolymers. Furthermore, the developed photonic pigments are responsive, with a selective and reversible color change observed upon swelling in different solvents. Our system is particularly suited for the scalable production of photonic pigments, arising from their rapid self-assembly mechanism and size-independent color.
\end{abstract}

\title{
Keywords
}

bottlebrush block copolymers, photonic crystals, confined self-assembly, structural colors, microspheres 
Hierarchical self-assembled materials that exhibit structural color are ideal candidates for photonic pigments, as they offer the possibility to manipulate the visual appearance in terms of both the color and the scattering response. ${ }^{1,2}$ Such effects are also found in nature, where vivid and metallic colors are often the result of intricate nano- and micro-scale structural motifs. ${ }^{3-5}$ Recently, the fabrication of photonic pigments from hierarchically-assembled photonic materials has been demonstrated. Such systems are typically obtained by restricting the self-assembly of either colloidal particles ${ }^{6-14}$ or liquid crystals ${ }^{15-17}$ within a confined geometry, such as a micron-sized droplet. However, despite being extensively studied, these systems usually have strong intrinsic limitations, especially in terms of formulation and scalability, which are key for real-world applications. Colloidal photonic structures, for instance, exhibit a color that is typically dependent on their porosity (that can be lost due to refractive index matching in formulation) and polydispersity (which causes incoherent scattering). Additionally, the reflectivity of cholesteric liquid crystal systems is theoretically limited to $50 \%$ and this coloration is challenging to preserve in the solid-state.

Block copolymers provide a promising alternative for bottom-up fabrication of photonic structures. ${ }^{18-22}$ In particular, bottlebrush block copolymers (BBCPs), where a highly extended backbone is densely grafted with polymer branches, are well-suited for photonic pigments as they can form lamellar structures with domain spacings large enough to produce a strong reflection in the visible spectrum. ${ }^{23-29}$ However, to date, the photonic properties of BBCPs have mainly been investigated in the film geometry. Here we report the confinement of BBCPs within spherical microdroplets to produce hierarchical photonic pigments and demonstrate that this confinement leads to unprecedented long-range ordering of the lamellae, resulting in up to $100 \%$ reflectivity. 


\section{Results and Discussion}

To demonstrate this strategy, the bottlebrush block copolymer (poly(norbornene)-graftpoly(styrene))-block-(poly(norbornene)-graft-poly(dimethylsiloxane)) was employed, although it should be noted that the concept can be generalized to other BBCP systems. This BBCP was synthesized using sequential ring opening metathesis polymerization (ROMP) with precise control over the molecular weight (MW) and the backbone length. Three representative samples (BBCP1-3) were prepared with number average MW $\left(\mathrm{M}_{\mathrm{n}}\right)$ of 3.3, 4.1 and 7.1 MDa respectively (Figure S1-2, Supporting Information). As a preliminary test, the photonic properties of the three BBCPs were confirmed by casting films from a $20 \mathrm{mg} / \mathrm{mL}$ chloroform solution. The dry films were observed to visibly reflect color at specular angles, ranging from blue to red upon increasing the MW of the constituent BBCPs, with cross-sectional scanning electron microscopy (SEM) confirming the lamellar structure (Figure S3, Supporting Information). ${ }^{28}$

To produce hierarchical photonic pigments, the BBCPs were geometrically-confined within micron-scale droplets which, upon loss of solvent, yielded spherical polymer microparticles ('microspheres'). Monodisperse microdroplets (diameter, $\varnothing \approx 160 \mu \mathrm{m}$ ) were generated in a single step within a flow-focusing microfluidic device as a chloroform-in-water emulsion with polyvinyl alcohol as the aqueous surfactant, as described in the Experimental Methods and Figure S4 (Supporting Information). As the chloroform droplets were denser than the surrounding aqueous phase, they settled onto the substrate, with the thickness of the water layer used to control the drying rate. Upon complete loss of chloroform, vivid, monochromatic microspheres $(\varnothing \approx 45 \mu \mathrm{m})$ were formed as a suspension in water. As with the BBCP films, the trend in reflected color 
correlates with the $\mathrm{MW}$ of the constituent $\mathrm{BBCP},{ }^{28}$ with blue, green and red iridescent microspheres prepared (Figure 1b and Figure S5, Supporting Information).

Optical microscopy on a single microsphere revealed a brightly colored reflection (Figure 1c and Figure S6a, Supporting Information), with an associated corresponding sharp peak in the reflectance spectrum (Figure 1d and Figure S7, Supporting Information). This intense reflection, up to $100 \%$ reflectivity relative to a silver mirror, is attributed to the formation of a highly-ordered multi-layer lamellar structure within the microsphere, as shown schematically in Figure 1a. This curved multi-layer structure interacts with light following Bragg's law, resulting in a central reflectance spot (with dimensions defined by the numerical aperture of the objective) ${ }^{10}$ and, when in close proximity to neighboring microspheres, blue-shifted secondary spots arising from crosscommunication (Figure S8, Supporting Information). ${ }^{15-17}$ The presence of a concentric lamellar structure was confirmed by SEM, where rings are clearly observed through the entire cross-section (Figure 1e).

Observation of individual microspheres of BBCP1-3 between crossed polarizers revealed strong birefringence with a characteristic Maltese cross (Figure S6a, Supporting Information). This indicates extensive long-range ordering at the molecular level of the (birefringent) polystyrene bristles within a concentric lamellar structure. Notably, for a comparable drying time the intensity of the birefringence decreased from BBCP1 to BBCP3, with an increasingly significant dark region observed at the center of each microsphere. This observation suggests the onset of kinetic arrest upon drying can inhibit the reorientation of domains formed from longer, less mobile molecular brushes - in agreement with our previous study. ${ }^{30}$ 
The evolution of structural color was monitored in real-time and correlated to the self-assembly process. As shown in Figure S9 (Supporting Information), droplets of BBCP1 were observed to develop a violet spot at the center upon reaching a critical concentration $(\sim 120 \mathrm{mg} / \mathrm{mL})$, corresponding to the formation of concentric lamellae, irrespective of drying rate. Upon further concentration, this violet spot first underwent a color-shift to green (due to loss of chloroform increasing the average refractive index), followed by a shift back to blue upon final loss of solvent (due to compression of the lamellae). The onset of birefringence was also observed at this critical concentration, first near the droplet interface and progressively extending towards the center as the concentration increased (Figure S10, Supporting Information). This indicates that the liquid-liquid droplet interface directs the assembly of the lamellar structure, promoting long-range concentric ordering and ensuring high reflectance from the microsphere by restricting any defects or disorder to the core of the microsphere. This mechanism also suggests that smaller microspheres, where the surface-to-volume ratio is greater, should more readily form a well-ordered concentric lamellar structure (Figure S11, Supporting Information). However, it should be noted that an axiallystacked lamellar morphology was instead reported for BBCP nano-emulsions, ${ }^{31}$ where the concentric lamellar structure is highly disfavored by the significantly greater curvature of a nanodroplet $(\varnothing<<1 \mu \mathrm{m})$. To understand the role of the surfactant on this interfacial-directed selfassembly process, BBCP1 microspheres were also prepared using ionic, amphiphilic surfactants: sodium dodecyl sulfate (SDS) and cetrimonium bromide (CTAB). Instead of a concentric lamellar structure, the microspheres exhibited a disordered, red-shifted and speckled appearance with a correspondingly weak reflectance of $<10 \%$ (Figure S12a, Supporting Information). This confirms that the interaction of the $\mathrm{BBCP}$ with the liquid-liquid interface is critical to promote the initial 
growth of the concentric lamellae, as achieved only when the non-ionic polymeric stabilizer PVA was used as the surfactant.

The obtained color can be further tuned by producing microdroplets containing binary polymer blends $^{25}$ of BBCP1 to BBCP3 (Figure 2a and Figure S6b, Supporting Information). The color of the blended microspheres lies between that expected for the two constituent BBCPs, with e.g. a 1:1 mixture of BBCP1 ('blue') and BBCP3 ('red') resulting in well-ordered, green microspheres. This is noteworthy, as it demonstrates that the desired color can be achieved through the formulation of the initial $\mathrm{BBCP}$ solution, rather than through meticulous control of the polymer MW. Furthermore, the intensity of the reflection is not compromised by using a binary blend (Figure $2 \mathrm{~b}$ ), suggesting that a broad spectrum of photonic pigments can be readily prepared.

Since the color reflected from a microsphere depends on the periodicity of the lamellae, the color is responsive to swelling in specific solvents. As shown in Figure 2c, microspheres of BBCP1 were observed to dramatically shift from blue, through green to red upon immersion in ethanol, cyclohexanol and tert-butanol respectively. This color change can be attributed to selective swelling of the poly(dimethylsiloxane) domains, with the degree of swelling correlating with the reduction in polarity of the alcohols (respectively $0.65,0.51$ and 0.39 relative to water ${ }^{32}$ ). This swelling-induced color change is reversible, with the original color being restored upon drying. This trend is similarly observed for BBCP2-3 (Figure 2d), where interestingly the $2^{\text {nd }}$ order reflectance peaks shift into the visible spectrum when immersed in tert-butanol (Figure S13, Supporting Information). 
BBCP films are known to be highly tolerant to the inclusion of additives that can impart additional functionality. ${ }^{30,33-35}$ This property was exploited here to reduce the intrinsic multiple scattering induced by the small dimensions of the microspheres. Through the addition of carbon black, a broadband absorber, the vividness of the structural color could be enhanced with negligible disturbance on the self-assembly process (Figure 2e-f). The BBCP photonic pigments are also highly tolerant against heating up to $\sim 170{ }^{\circ} \mathrm{C}$, above which the color is rapidly lost. Below this temperature, the bright reflection spot is maintained, with a deformation-induced red-shift observed between $140-170{ }^{\circ} \mathrm{C}$ (Figure S14, Supporting Information).

To understand the robustness and the scalability of the fabrication process, the role of the initial BBCP concentration, droplet size and the drying rate were explored. Figure 3a-b shows that comparable structurally-colored microspheres were formed for all investigated timescales (from a few minutes to a day), with longer drying times leading to slightly higher reflectance and less observable defects. This suggests that the self-assembly of BBCP is rapid, benefiting from greatly reduced polymer chain entanglement relative to their linear analogs. ${ }^{30}$ To investigate the role of the initial BBCP concentration, droplets were prepared from a $140 \mathrm{mg} / \mathrm{mL}$ BBCP1 solution above the critical concentration for self-assembly. As shown in Figure S12b (Supporting Information), photonic pigments prepared at this higher initial concentration can be formed, however there is a significant degree of disorder within the structure that severely impacts the optical appearance, giving rise to a much dimmer central blue spot ( $<30 \%$ reflection) and weak, green patches originating from kinetically-trapped lamellae. In contrast, vibrant photonic microspheres have been successfully prepared from an initial concentration of $20-40 \mathrm{mg} / \mathrm{mL}$. This suggests that the initial $\mathrm{BBCP}$ concentration can be increased up to the critical concentration 
without affecting the optical properties of the final photonic pigment. To explore the role of size on the optical properties, highly polydisperse microspheres were prepared by vortex-assisted emulsification, as described in the Experimental Methods. As shown in Figure 3c, the reflected color and consequently the underlying nanostructure of the microspheres is not affected by the size of the templating microdroplet. Simulation of a one-dimensional Bragg reflector of BBCP1 predicted $100 \%$ reflectance for a film comprising $36+$ bilayers, corresponding to a $\geq 5.9 \mu \mathrm{m}$ thick film (Figure 3d: solid line). Consequently, it is expected that well-ordered microspheres of $6 \mu \mathrm{m}$ diameter or above will reflect $100 \%$ of incident light in the photonic band gap. However, the measured reflected intensity of single BBCP1 microspheres appears to decrease significantly below $40 \mu \mathrm{m}$ (Figure 3d: circles). This reduction is an artifact of the experimental microscope setup and is attributed to the geometrical limitation of interrogating a single microsphere with a large numerical aperture objective (see Experimental Methods and Figure 3d: dashed lines). As the measured value for large microspheres $(40+\mu \mathrm{m})$ are in good agreement with the 1D simulation, we believe that the reflectivity of well-ordered microspheres with dimensions of 6-40 $\mu \mathrm{m}$ should also have a comparably high reflectivity of $\sim 100 \%$.

To demonstrate the viability of the developed photonic pigment and to evaluate the consequences of its hierarchical structure, the microspheres were embedded within a transparent matrix. Only 2 wt.\% of polydisperse BBCP2 microspheres was required to give a visibly green and metallic coloration to a $\sim 20 \mathrm{~cm}^{2}$ cross-linked PDMS film (for fabrication see Experimental Methods). The concentric lamellar structure (in contrast to a planar geometry) provides a uniform green color under diffuse illumination, with no evident iridescence (Figure 4a). However, when under direct illumination the film exhibits strong iridescence, with a blue-shift observed at large angles $\left(\theta>30^{\circ}\right.$, 
Figure 4b). The advantages of the microsphere-embedded film over the equivalent drop-cast BBCP film (Figure S3, Supporting Information) are the much-improved material efficiency and the desirable non-iridescent appearance. Furthermore, this ability to simply mix the photonic pigments into the polymer precursor solution will allow for them to be readily coated onto complex 3-D surfaces ( $c f$. mica-based interference pigments in automotive paint).

\section{Conclusions}

In summary, we demonstrate a scalable approach for the fabrication of photonic pigments via the confined self-assembly of bottlebrush block copolymers. The obtained microspheres show strong, sharp reflectance peaks, with tunable and responsive colors, arising from the well-ordered concentric multi-layer photonic structure. The self-assembly process is robust, with high tolerance to the initial droplet geometry and the drying rate. Furthermore, the strong templating effect of the droplet interface, combined with the high surface-to-volume ratio enhances long-range ordering leading to high reflectivity. As such, $\mathrm{BBCP}$ microspheres have great potential as photonic pigments to be used as a replacement for colorants in e.g. automotive paints, or in advanced photonic devices.

\section{Experimental Methods}

Synthesis of Norbornene-terminated polystyrene (PS-NB). Hydroxy-terminated polystyrene (PS-OH) was synthesized according to the reported method. ${ }^{36}$ A mixture of styrene $(20 \mathrm{~mL}, 174$ mmol), copper (I) bromide (0.29 g, $2.0 \mathrm{mmol})$, 2-hydroxyethyl 2-bromoisobutyrate $(0.42 \mathrm{~g}, 2.0$ $\mathrm{mmol})$, and toluene $(2.2 \mathrm{~mL})$ in a $100 \mathrm{~mL}$ Schlenk flask was subjected to three freeze-pump-thaw cycles. The flask was placed in an oil bath preheated to $90{ }^{\circ} \mathrm{C}$, and $\mathrm{N}, \mathrm{N}, \mathrm{N}$, $\mathrm{N}$ ', $\mathrm{N}$ ' pentamethyldiethylenetriamine (PMDETA, $0.42 \mathrm{~mL}, 2.0 \mathrm{mmol}$ ) was injected via a 
nitrogen-purged syringe. After 70 min, the flask was cooled in liquid nitrogen, diluted with tetrahydrofuran (THF), and passed through a neutral alumina column to remove the copper catalyst. The absorbent in the column was washed with THF $(30-40 \mathrm{~mL})$ and the resulting polymer solution was concentrated by rotary evaporation. The polymer was precipitated in methanol and dried under vacuum $\left(M_{\mathrm{n}}=4.7 \mathrm{kDa}\right.$ from ${ }^{1} \mathrm{H}-\mathrm{NMR}$ analysis $)$.

An esterification reaction was employed in the preparation of norbornene-terminated polystyrene (PS-NB). Exo-5-norbornenecarboxylic acid (0.19 g, $1.4 \mathrm{mmol})$, PS-OH (4.2 g, $0.91 \mathrm{mmol})$ and N,N'-dicyclohexyl-carbodiimide (DCC, $0.23 \mathrm{~g}, 1.1 \mathrm{mmol}$ ) were added into a $100 \mathrm{~mL}$ of Schlenk flask followed by dichloromethane (DCM, $30 \mathrm{~mL}$, anhydrous). The resulting solution was put in an ice bath for $30 \mathrm{~min}$ before adding 4 dimethylaminopyridine (DMAP, $12 \mathrm{mg}, 0.10 \mathrm{mmol}$ ). The reaction mixture was stirred at room temperature for approx. $8 \mathrm{~h}$, filtered and poured into cold methanol to yield the product as a white solid. Two more precipitations in methanol were carried out to remove excess exo-5-norbornenecarboxylic acid.

Synthesis of Norbornene-terminated poly(dimethylsiloxane) (PDMS-NB). Under nitrogen flow, monohydroxy-terminated poly(dimethylsiloxane) $\left(M_{\mathrm{n}} \approx 4.67 \mathrm{kDa}, 14 \mathrm{~g}, 3.0 \mathrm{mmol}\right)$, exo-5norbornenecarboxylic acid $(0.62 \mathrm{~g}, 4.5 \mathrm{mmol})$, and DCC ( $0.93 \mathrm{~g}, 4.5 \mathrm{mmol})$ were added into a 100 $\mathrm{mL}$ of Schlenk flask followed by DCM (45 mL, anhydrous). The resulting solution was put in an ice bath for $30 \mathrm{~min}$ before adding DMAP $(12 \mathrm{mg}, 0.10 \mathrm{mmol})$. The reaction mixture was stirred at room temperature for approx. $48 \mathrm{~h}$, filtered to remove precipitates, and washed with aqueous sodium hydroxide solution $(0.1 \mathrm{M})$ to give the product as a colorless liquid. 
General procedure for block copolymerization of two macromonomers via Ring Opening Metathesis Polymerization (ROMP). ${ }^{28,37}$ In a typical experiment, $200 \mathrm{mg}$ of each macromonomer were added to separate Schlenk tubes followed by the desired amount of anhydrous DCM such that the concentration of the macromonomer was between $0.05-0.1 \mathrm{M}$. The resulting solutions were degassed by three freeze-pump-thaw cycles. The polymerization of PS-NB was initiated at room temperature by adding the desired amount of the $3^{\text {rd }}$ generation Grubbs catalyst solution in DCM. When the first macromonomer (PDMS-NB) had polymerized the solution of the second macromonomer (PS-NB) was added to the reaction mixture. This solution was stirred for an additional 2-3 hours, before quenching with ethyl vinyl ether. The conversion rate of PS-NB and PDMS-NB were approx. $100 \%$ based on ${ }^{1} \mathrm{H}-\mathrm{NMR}$ spectroscopy.

Solution formulation. The bottlebrush block copolymers (BBCP1-3, $20 \mathrm{mg}$ ) was added to a vial followed by chloroform $(1.0 \mathrm{~mL})$ affording a stock solution $(20 \mathrm{mg} / \mathrm{mL})$. Blends of BBCP1-3 were prepared by mixing the stock solutions, such that the overall concentration remained 20 $\mathrm{mg} / \mathrm{mL}$ in chloroform. For the aqueous phase, poly(vinyl alcohol) (PVA, $200 \mathrm{mg}$ ) was employed as a surfactant and as such was dissolved with heating into Milli-Q water $(10 \mathrm{~mL})$ to form a clear solution. Aqueous solutions of SDS and CTAB were prepared analogously at a concentration of $20 \mathrm{mg} / \mathrm{mL}$. The solutions were filtered to remove dust before use. Where used, carbon black was dispersed into chloroform as a $50 \mu \mathrm{g} / \mathrm{mL}$ suspension using bath sonication prior to addition of 20 $\mathrm{mg} / \mathrm{mL}$ of BBCP1.

Droplet-based microfluidic emulsification. Monodisperse microdroplets were generated within a hydrophilic, etched-glass microfluidic device containing a $105 \mu \mathrm{m}$ wide flow-focusing junction 
and a channel depth of $100 \mu \mathrm{m}$ (Dolomite \#3000158). To generate chloroform-in-water droplets, the aqueous PVA solution and the immiscible BBCP solution in chloroform were injected into the microfluidic device via two syringe pumps (Harvard Apparatus, PHD 2000) with controlled flow rates of 200 and $150 \mu \mathrm{L} \cdot \mathrm{h}^{-1}$ respectively. At the intersection, the shear forces caused the formation of droplets of chloroform, surrounded by water (typically $\varnothing \approx 160 \mu \mathrm{m}$ ). Once the micro-emulsion was generated, the droplets exited the microfluidic device through microbore polythene tubing $\left(\varnothing_{I}\right.$ $=380 \mu \mathrm{m}, 1 \approx 8 \mathrm{~cm}$ ) and were collected into a water-filled glass Petri dish (Milli-Q) and covered to slow evaporation. This allowed the loss of chloroform to be reduced such that the drying time could be varied between minutes (i.e. 1-3 mm depth of water, Petri dish open to ambient conditions) to days (i.e. 1-2 cm depth of water, covered Petri dish). In all cases, discrete polymer microspheres were formed upon loss of chloroform, with no evidence of droplet fusion, substrate wetting, or any other forms of instability recorded during this process. Dried microspheres (typically $\varnothing \approx 45 \mu \mathrm{m}$ ) were washed with Milli-Q water to remove residual PVA surfactant. Furthermore, it was observed that aqueous dispersions of $\mathrm{BBCP}$ microspheres show no evidence of degradation over the course of a year, and such dispersions can be repeatably dried to a powder and redispersed into a non-swelling solvent (e.g. water, methanol) with mild bath sonication.

Vortex-assisted emulsification. Typically, the immiscible aqueous PVA solution $(6.0 \mathrm{~mL})$ and the chloroform BBCP solution $(1.0 \mathrm{~mL})$ were combined in a $10 \mathrm{~mL}$ screw cap vial. A highly polydisperse emulsion of chloroform microdroplets was then simply created by inducing a vortex for one minute (Gallenkamp Spinmix SGP-205-010X). The obtained droplets were transferred immediately into a glass petri dish filled with an appropriate amount of Milli-Q water and dried as described above. It is important to note that vortex-assisted emulsification produces chemically- 
identical microdroplets to microfluidic emulsification. Furthermore, the optical response and visual appearance of comparably-sized microspheres dried under equivalent conditions is indistinguishable with respect to the emulsification method used.

$B B C P$ films. Thin films of BBCP1-3 were prepared by drop-casting a $20 \mathrm{mg} / \mathrm{mL}$ solution in chloroform onto a glass slide and allowing it to evaporate slowly over $24 \mathrm{~h}$ for BBCP1-2 or $48 \mathrm{~h}$ for BBCP3. The resultant films visibly reflected color at specular angles, with a clear red-shift upon increasing the molecular weight from BBCP1 to BBCP3.

BBCP microsphere-embedded films. PDMS and the cross-linker (Sylgard 184 elastomer kit) were mixed in a 10:1 ratio, prior to blending with a concentrated solution of BBCP2 microspheres in methanol. Air bubbles and residual solvent were then removed from the mixture under reduced pressure to yield a transparent liquid containing $2 \mathrm{wt} . \%$ of visibly green microspheres. The mixture was poured onto a black cardboard substrate and allowed to cure at room temperature for approx. $2 \mathrm{~h}$. No swelling of the microspheres was observed in PDMS.

Simulation. The interaction of light with BBCP microspheres was simulated using Lumerical FDTD Solutions (Lumerical Inc.), which is based on the three-dimensional finite-difference timedomain (FDTD) method. ${ }^{38}$ For the 1D multilayer, the incident light beam was a plane wave with a broadband spectrum in the range $300-700 \mathrm{~nm}$. The boundary conditions were set as absorbing in the direction of the light propagation and as periodic in the perpendicular direction. The BBCP structure was constructed as a flat multilayer stack with alternating layers of PS and PDMS, with PDMS as the outermost layer. The wavelength-dependent refractive index for the PS and PDMS 
layers was obtained from ellipsometry measurements of individual PS and PDMS macromonomer films (Figure S15a, Supporting Information). The thickness of the PS and of the PDMS domains were estimated as $0.08 \mu \mathrm{m}$ and $0.084 \mu \mathrm{m}$ for BBCP1, as inferred from the SEM cross-sections of the film and the volume fraction of the $\mathrm{BBCP}$. For the 3D microsphere, the incident light beam was a Gaussian wave with a broadband spectrum in the range $300-900 \mathrm{~nm}$, using the thin lens approximation, with the numerical aperture set to match the experimental microscopy configuration $(\mathrm{NA}=0.55)$ and the boundary conditions were set as absorbing (Figure S15c, Supporting Information). The BBCP structure was constructed as a concentric multilayer sphere with alternating layers of PS and PDMS, as above. For both the 1D and the 3D model the reflectance was calculated as the ratio of the light flux incident on the structure and the light flux reflected through the detector plane (Figure S15b,d, Supporting Information).

The reduction in the measured reflectance intensity, when compared to the 1D simulation, arises from the experimental constraints of interrogating individual microspheres (radius: $r_{\text {sphere }}$ ) with a microscope, as shown schematically in Figure S16 (Supporting Information). A highmagnification and numerical aperture (NA) objective is a requirement for the correct imaging and micro-spectroscopy of small microparticles. However, as the NA of collection and illumination were fixed, a proportion of the light is lost due to the curvature of the spherical microparticle. A first-order approximation of this effect was geometrically-calculated by considering the area ratio between two cones of reflectance at the working distance of the objective $(9 \mathrm{~mm})$. The first cone was described by the maximum NA of the objective (0.55), while the second larger cone was geometrically-corrected for the maximum curvature of the surface of the microsphere within the region collected by the optical fiber (approximated to a circle of radius: $r_{\text {fibre }}=0.95 \mu \mathrm{m}$ ). Using 
the normalized ratio described in Equation 1, the 1D simulation was recalculated to account for the proportion of light reflected from $\mathrm{BBCP}$ microspheres that could not be collected by the spectrometer.

$$
\text { ratio }=\left(\frac{\tan \left(\sin ^{-1}(\mathrm{NA})\right)}{\tan \left(\sin ^{-1}(\mathrm{NA})+\sin ^{-1}\left(\frac{r_{\text {fibre }}}{r_{\text {sphere }}}\right)\right)}\right)^{2}
$$

\section{Supporting Information}

Supporting Figures and further experimental methods are available free of charge via the Internet at http://pubs.acs.org. Additional data relating to this publication is available from the University of Cambridge data repository (https://doi.org/10.17863/CAM.32022).

\section{Author Information}

Dr. D.-P. Song*

Tianjin Key Laboratory of Composite and Functional Materials, School of Materials Science and Engineering, Tianjin University, Tianjin 300350, People's Republic of China.

* E-mail: dpsong33@gmail.com

T. H. Zhao, Dr. G. Guidetti, Dr. S. Vignolini, * Dr. R. M. Parker

Department of Chemistry, University of Cambridge, Lensfield Road, Cambridge, CB2 1EW, UK.

* Email: sv319@cam.ac.uk

\section{Author Contributions}


${ }^{\dagger}$ Dong-Po Song and Tianheng H. Zhao contributed equally. The authors declare no competing financial interest.

\section{Funding sources}

This work was supported by the European Research Council [ERC-2014-STG H2020 639088], the BBSRC [David Phillips Fellowship BB/K014617/1], the EPSRC [1525292; EP/N016920/1; EP/R511675/1], the National Natural Science Foundation of China [Grant 51873098] and the Winton Programme for the Physics of Sustainability.

\section{Acknowledgements}

The authors would like to thank C. Abell for the generous use of his microfluidics research laboratory (Univ. Cambridge), B. Yavitt for GPC measurements (Univ. Massachusetts) and B. Frka-Petesic and G. Jacucci for valuable discussions (Univ. Cambridge).

\section{References}

(1) Zhao, Y.; Shang, L.; Cheng, Y.; Gu, Z. Spherical Colloidal Photonic Crystals. Acc. Chem. Res. 2014, 47, 3632-3642.

(2) Goerlitzer, E. S. A.; Klupp Taylor, R. N.; Vogel, N. Bioinspired Photonic Pigments from Colloidal Self-Assembly. Adv. Mater. 2018, 1706654.

(3) Fu, Y.; Tippets, C. A.; Donev, E. U.; Lopez, R. Structural Colors: From Natural to Artificial Systems. Wiley Interdiscip. Rev. Nanomedicine Nanobiotechnology 2016, 8, 758-775.

(4) Sun, J.; Bhushan, B.; Tong, J. Structural Coloration in Nature. RSC Adv. 2013, 3, 14862.

(5) Johansen, V. E.; Onelli, O. D.; Steiner, L. M.; Vignolini, S. Photonics in Nature: From Order to Disorder. In Functional Surfaces in Biology III: Diversity of the Physical 
Phenomena; Gorb, S. N., Gorb, E. V, Eds.; Springer International Publishing: Cham, 2017; pp 53-89.

(6) Yu, Z.; Wang, C.-F.; Ling, L.; Chen, L.; Chen, S. Triphase Microfluidic-Directed SelfAssembly: Anisotropic Colloidal Photonic Crystal Supraparticles and Multicolor Patterns Made Easy. Angew. Chemie Int. Ed. 2012, 51, 2375-2378.

(7) Zhang, B.; Cheng, Y.; Wang, H.; Ye, B.; Shang, L.; Zhao, Y.; Gu, Z. Multifunctional Inverse Opal Particles for Drug Delivery and Monitoring. Nanoscale 2015, 7, 10590_ 10594.

(8) Zhao, Y.; Xie, Z.; Gu, H.; Jin, L.; Zhao, X.; Wang, B.; Gu, Z. Multifunctional Photonic Crystal Barcodes from Microfluidics. NPG Asia Mater. 2012, 4, e25-7.

(9) Zhao, Y.; Zhao, X.; Hu, J.; Xu, M.; Zhao, W.; Sun, L.; Zhu, C.; Xu, H.; Gu, Z. Encoded Porous Beads for Label-Free Multiplex Detection of Tumor Markers. Adv. Mater. 2009, $21,569-572$.

(10) Vogel, N.; Utech, S.; England, G. T.; Shirman, T.; Phillips, K. R.; Koay, N.; Burgess, I. B.; Kolle, M.; Weitz, D. A.; Aizenberg, J. Color from Hierarchy: Diverse Optical Properties of Micron-Sized Spherical Colloidal Assemblies. Proc. Natl. Acad. Sci. 2015, $112,10845-10850$.

(11) Park, J. G.; Kim, S. H.; Magkiriadou, S.; Choi, T. M.; Kim, Y. S.; Manoharan, V. N. FullSpectrum Photonic Pigments with Non-Iridescent Structural Colors through Colloidal Assembly. Angew. Chemie - Int. Ed. 2014, 53, 2899-2903.

(12) Kim, S. H.; Park, J. G.; Choi, T. M.; Manoharan, V. N.; Weitz, D. A. Osmotic-PressureControlled Concentration of Colloidal Particles in Thin-Shelled Capsules. Nat. Commun. 2014, 5, 3068.

(13) Xiao, M.; Hu, Z.; Wang, Z.; Li, Y.; Tormo, A. D.; Le Thomas, N.; Wang, B.; Gianneschi, N. C.; Shawkey, M. D.; Dhinojwala, A. Bioinspired Bright Noniridescent Photonic Melanin Supraballs. Sci. Adv. 2017, 3, e1701151.

(14) Lan, Y.; Caciagli, A.; Guidetti, G.; Yu, Z.; Liu, J.; Johansen, V. E.; Kamp, M.; Abell, C.; Vignolini, S.; Scherman, O.; Eiser, E. Unexpected Stability of Aqueous Dispersions of Raspberry-like Colloids. Nat. Commun. 2018, 9, 3614. 
(15) Geng, Y.; Noh, J.; Drevensek-Olenik, I.; Rupp, R.; Lenzini, G.; Lagerwall, J. P. F. HighFidelity Spherical Cholesteric Liquid Crystal Bragg Reflectors Generating Unclonable Patterns for Secure Authentication. Sci. Rep. 2016, 6, 2-10.

(16) Lee, S. S.; Kim, S. K.; Won, J. C.; Kim, Y. H.; Kim, S. H. Reconfigurable Photonic Capsules Containing Cholesteric Liquid Crystals with Planar Alignment. Angew. Chemie Int. Ed. 2015, 54, 15266-15270.

(17) Noh, J.; Liang, H.-L.; Drevensek-Olenik, I.; Lagerwall, J. P. F. Tuneable Multicoloured Patterns from Photonic Cross-Communication between Cholesteric Liquid Crystal Droplets. J. Mater. Chem. C 2014, 2, 806-810.

(18) Stefik, M.; Guldin, S.; Vignolini, S.; Wiesner, U.; Steiner, U. Block Copolymer SelfAssembly for Nanophotonics. Chem. Soc. Rev. 2015, 44, 5076-5091.

(19) Lee, J.-H.; Koh, C. Y.; Singer, J. P.; Jeon, S.-J.; Maldovan, M.; Stein, O.; Thomas, E. L. Ordered Polymer Structures for the Engineering of Photons and Phonons. Adv. Mater. 2014, 26, 532-569.

(20) Valkama, S.; Kosonen, H.; Ruokolainen, J.; Haatainen, T.; Torkkeli, M.; Serimaa, R.; Brinke, G. Ten; Ikkala, O. Self-Assembled Polymeric Solid Films with TemperatureInduced Large and Reversible Photonic-Bandgap Switching. Nat. Mater. 2004, 3, 872876.

(21) Kang, Y.; Walish, J. J.; Gorishnyy, T.; Thomas, E. L. Broad-Wavelength-Range Chemically Tunable Block-Copolymer Photonic Gels. Nat. Mater. 2007, 6, 957-960.

(22) Yang, Y.; Kim, H.; Xu, J.; Hwang, M. S.; Tian, D.; Wang, K.; Zhang, L.; Liao, Y.; Park, H. G.; Yi, G. R.; Xie, X.; Zhu, J. Responsive Block Copolymer Photonic Microspheres. Adv. Mater. 2018, 30, 1707344.

(23) Rzayev, J. Molecular Bottlebrushes: New Opportunities in Nanomaterials Fabrication. ACS Macro Lett. 2012, 1, 1146-1149.

(24) Liberman-Martin, A. L.; Chu, C. K.; Grubbs, R. H. Application of Bottlebrush Block Copolymers as Photonic Crystals. Macromol. Rapid Commun. 2017, 38, 1700058.

(25) Miyake, G. M.; Piunova, V. A.; Weitekamp, R. A.; Grubbs, R. H. Precisely Tunable 
Photonic Crystals From Rapidly Self-Assembling Brush Block Copolymer Blends. Angew. Chemie Int. Ed. 2012, 51, 11246-11248.

(26) Piunova, V. A.; Miyake, G. M.; Daeffler, C. S.; Weitekamp, R. A.; Grubbs, R. H. Highly Ordered Dielectric Mirrors via the Self-Assembly of Dendronized Block Copolymers. $J$. Am. Chem. Soc. 2013, 135, 15609-15616.

(27) Macfarlane, R. J.; Kim, B.; Lee, B.; Weitekamp, R. A.; Bates, C. M.; Lee, S. F.; Chang, A. B.; Delaney, K. T.; Fredrickson, G. H.; Atwater, H. A.; Grubbs, R. H. Improving Brush Polymer Infrared One-Dimensional Photonic Crystals via Linear Polymer Additives. J. Am. Chem. Soc. 2014, 136, 17374-17377.

(28) Sveinbjörnsson, B. R.; Weitekamp, R. A.; Miyake, G. M.; Xia, Y.; Atwater, H. A.; Grubbs, R. H. Rapid Self-Assembly of Brush Block Copolymers to Photonic Crystals. Proc. Natl. Acad. Sci. U. S. A. 2012, 109, 14332-14336.

(29) Boyle, B. M.; French, T. A.; Pearson, R. M.; McCarthy, B. G.; Miyake, G. M. Structural Color for Additive Manufacturing: 3D-Printed Photonic Crystals from Block Copolymers. ACS Nano 2017, 11, 3052-3058.

(30) Song, D. P.; Jacucci, G.; Dundar, F.; Naik, A.; Fei, H. F.; Vignolini, S.; Watkins, J. J. Photonic Resins: Designing Optical Appearance via Block Copolymer Self-Assembly. Macromolecules 2018, 51, 2395-2400.

(31) Steinhaus, A.; Pelras, T.; Chakroun, R.; Gröschel, A. H.; Müllner, M. Self-Assembly of Diblock Molecular Polymer Brushes in the Spherical Confinement of Nanoemulsion Droplets. Macromol. Rapid Commun. 2018, 39, 1800177.

(32) Reichardt, C.; Welton, T. Solvents and Solvent Effects in Organic Chemistry, 4th ed.; Wiley-VCH: Weinheim, 2011.

(33) Song, D.-P.; Li, C.; Colella, N. S.; Lu, X.; Lee, J.-H.; Watkins, J. J. Thermally Tunable Metallodielectric Photonic Crystals from the Self-Assembly of Brush Block Copolymers and Gold Nanoparticles. Adv. Opt. Mater. 2015, 3, 1169-1175.

(34) Song, D.-P.; Li, C.; Li, W.; Watkins, J. J. Block Copolymer Nanocomposites with High Refractive Index Contrast for One-Step Photonics. ACS Nano 2016, 10, 1216-1223. 
(35) Song, D. P.; Shahin, S.; Xie, W.; Mehravar, S.; Liu, X.; Li, C.; Norwood, R. A.; Lee, J. H.; Watkins, J. J. Directed Assembly of Quantum Dots Using Brush Block Copolymers for Well-Ordered Nonlinear Optical Nanocomposites. Macromolecules 2016, 49, 50685075 .

(36) Gao, H.; Matyjaszewski, K. Synthesis of Molecular Brushes by “Grafting onto" Method: Combination of ATRP and Click Reactions. J. Am. Chem. Soc. 2007, 129, 6633-6639.

(37) Xia, Y.; Olsen, B. D.; Kornfield, J. A.; Grubbs, R. H. Efficient Synthesis of Narrowly Dispersed Brush Copolymers and Study of Their Assemblies: The Importance of Side Chain Arrangement. J. Am. Chem. Soc. 2009, 131, 18525-18532.

(38) Yee, K. S. Numerical Solution of Initial Boundary Value Problems Involving Maxwell's Equations in Isotropic Media. IEEE Trans. Antennas Propag. 1966, 14, 302-307. 


\section{Figures}
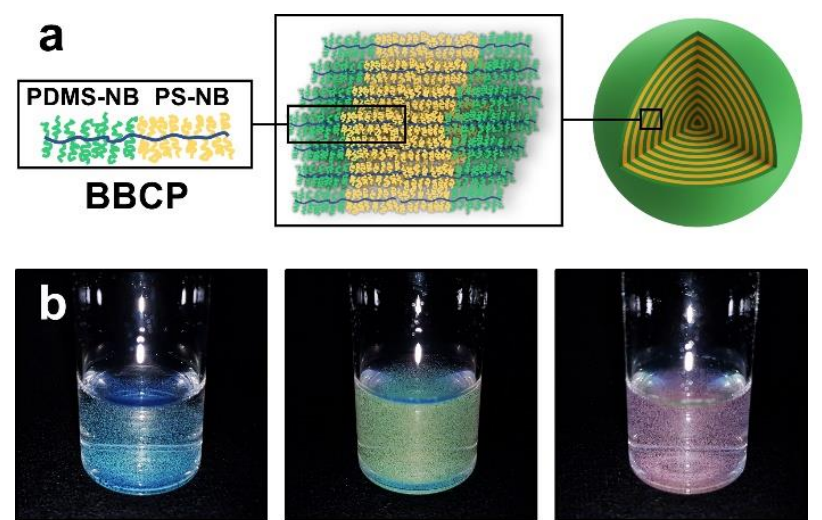

BBCP1
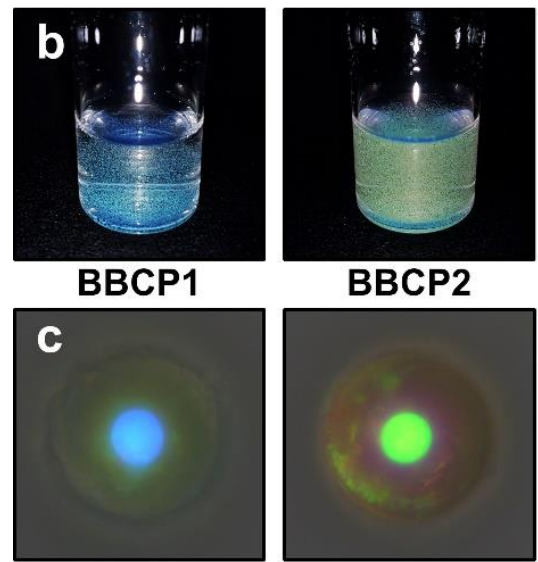

BBCP2
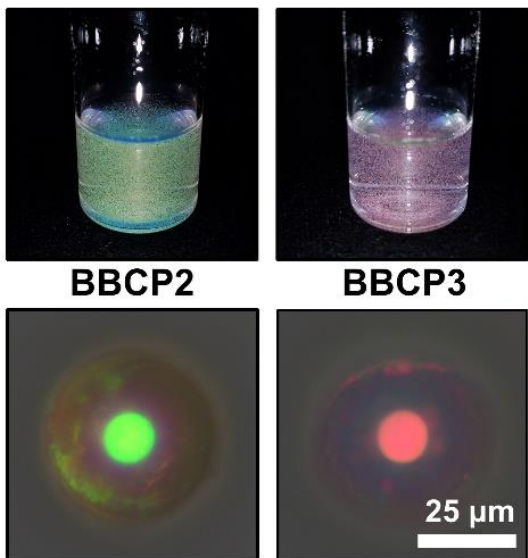

BBCP3

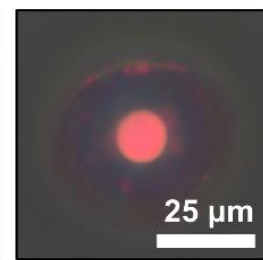

d
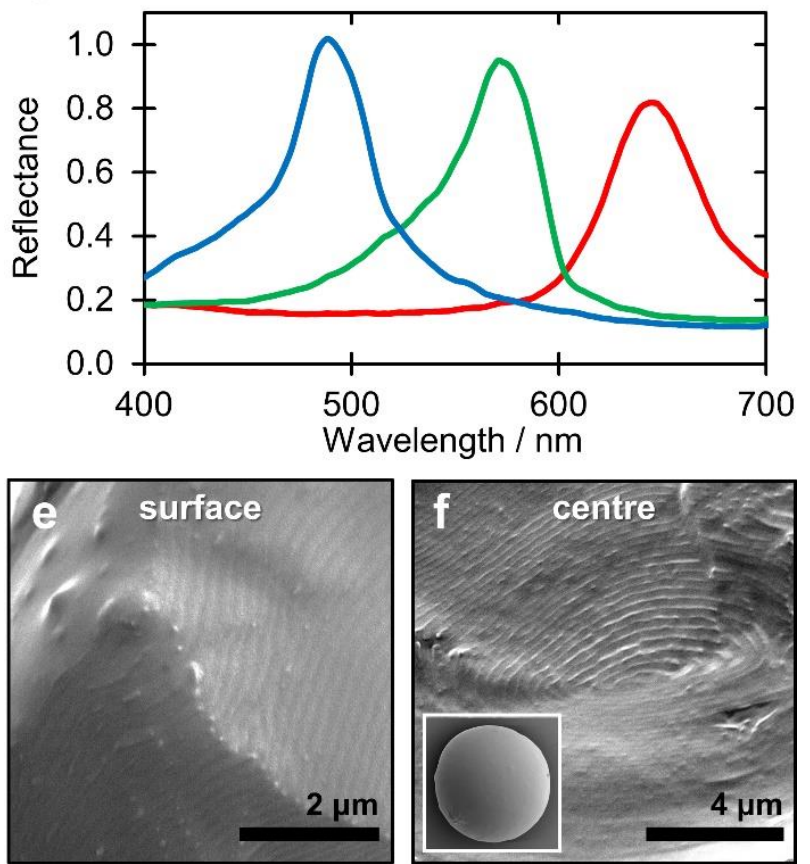

Figure 1. Photonic pigments of concentric, lamellar BBCP microspheres: (a) Schematic of the concentric lamellar structure formed inside a bottlebrush block copolymer (BBCP) microsphere. (b) Photograph showing BBCP1 (blue) BBCP2 (green) and BBCP3 (red) photonic pigments as a suspension in water, and (c) reflection micrographs of single microspheres. (d) Corresponding reflectance spectra for the microspheres shown in (c). (e-f) SEM cross-section of a BBCP1 microsphere, showing the lamellar structure extending from the (e) surface to the (f) center. The inset in (f) image shows an intact smooth microsphere $(\varnothing=45 \mu \mathrm{m})$. 

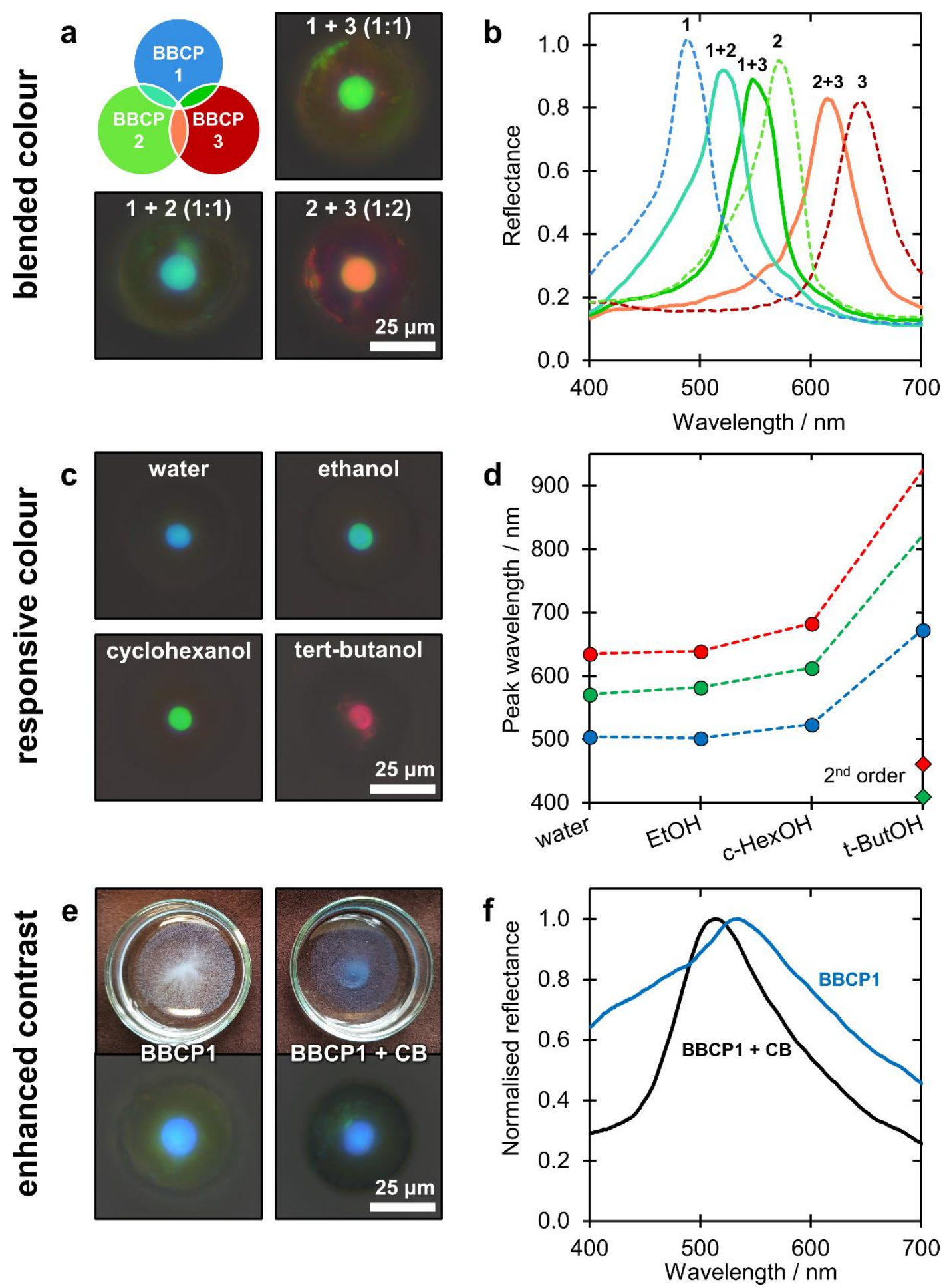

Figure 2. Tunable and responsive color from BBCP microspheres: (a) Micrographs and (b) corresponding reflectance spectra of polymer blends of BBCP1-3, showing the ability to produce a wide spectrum of secondary colors. (c) Micrographs of a BBCP1 microsphere illustrating the dramatic color change upon immersion in water, ethanol, cyclohexanol and tert-butanol. (d) A similar trend was observed for BBCP1 $(\bullet), \mathrm{BBCP} 2(\bullet)$ and BBCP3 $(\bullet)$ when plotted in terms of decreasing solvent polarity. In tert-butanol, $2^{\text {nd }}$ order peaks were instead observed in the visible 
range for BBCP2 $(\diamond)$ and BBCP3 $(\diamond)$ and these were used to extrapolate the $1^{\text {st }}$ order trend. (e) Photographs of Petri dishes $(\varnothing=35 \mathrm{~mm})$ containing microspheres of BBCP1 (left) and BBCP1 with $0.25 \mathrm{wt} \%$ carbon black (CB, right), demonstrating the improvement in contrast. (f) Large area reflectance spectra for the microsphere suspensions in (e) showing the reduced broadband scattering.
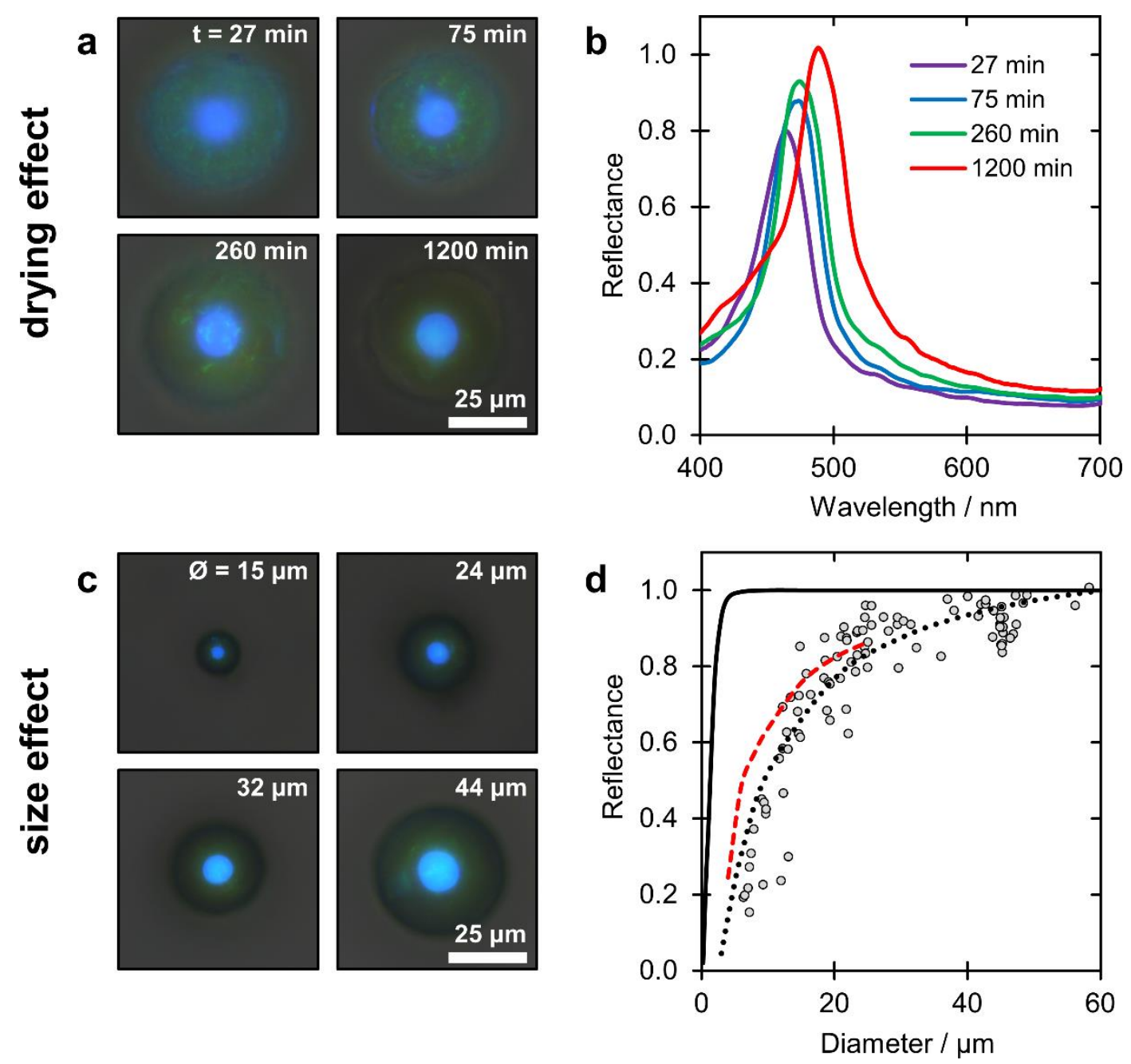

Figure 3. The impact of the fabrication process upon the optical properties of BBCP microspheres: (a) Micrographs and (b) reflectance spectra of BBCP1 microspheres dried over timescales from 27 minutes to 20 hours. (c) Micrographs of polydisperse microspheres of BBCP1 prepared by vortex emulsification. (d) The simulated peak reflectance of a 1D BBCP1 lamellar film saturates above $6 \mu \mathrm{m}$ thickness (-). In contrast, the measured intensity of single BBCP1 microspheres (O) shows a size dependence. However, correcting for probing a micron-scale sphere under fixed numerical aperture, using both a geometrically-corrected 1D model ( $\cdots)$ and a 3D spherical model (--), confirms this size dependence is an artefact of the geometrical constraint of the microscope set-up. 
a
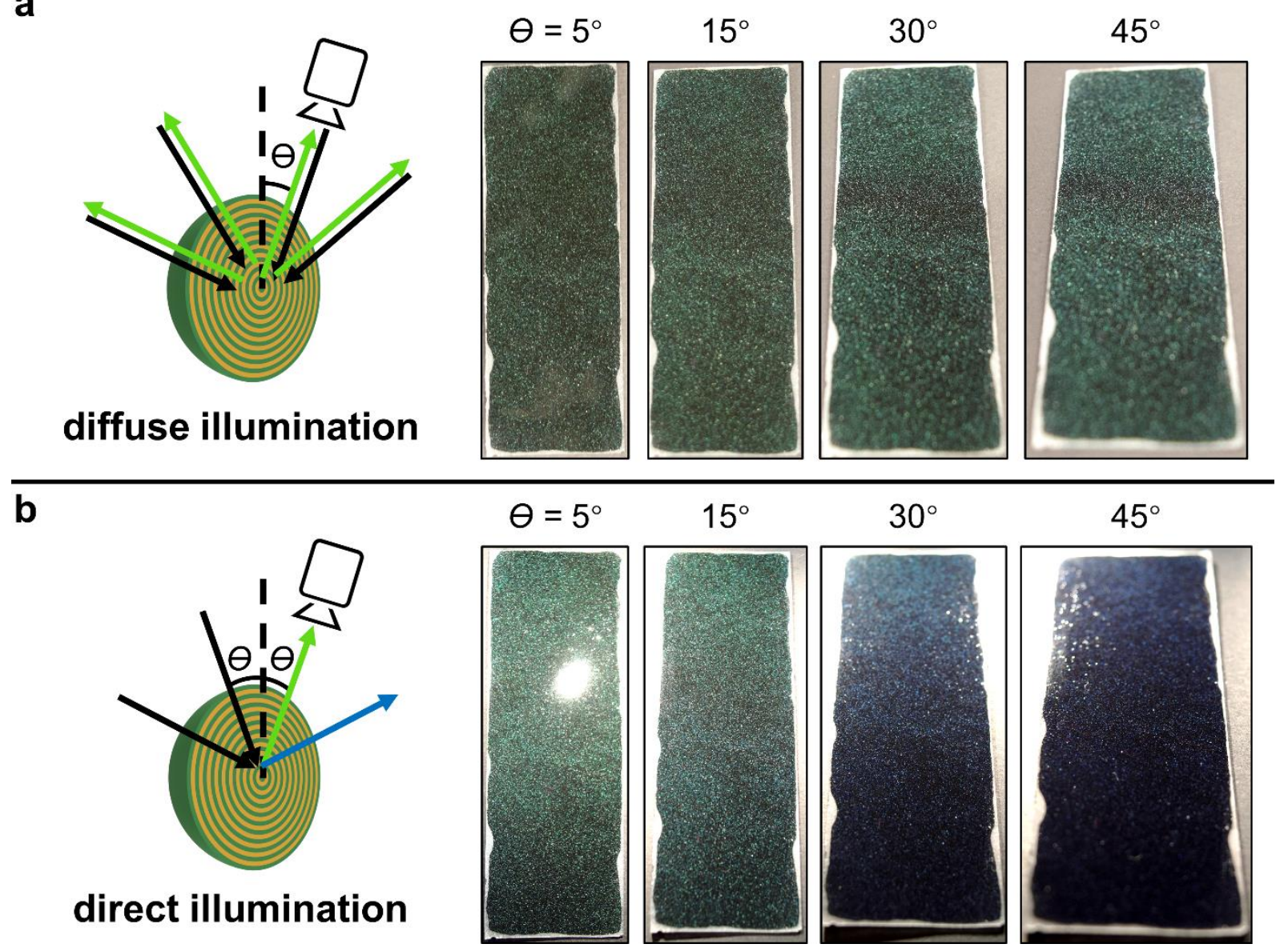

Figure 4. The effect of illumination upon the visual appearance of a microsphere-embedded film. (a-b) Photographs of a cross-linked PDMS film $(25 \times 75 \mathrm{~mm})$ containing approx. 2 wt.\% of BBCP2 microspheres as a photonic pigment: (a) under diffuse illumination the film appears green across a wide range of angles, and (b) under direct illumination the film is iridescent, with a blueshift upon increasing the angle between the light source and the observer. 
For Table of Contents

Hierarchical Photonic Pigments via the Confined Self-Assembly of Bottlebrush Block Copolymers

D.-P. Song*, T. H. Zhao, G. Guidetti, S. Vignolini* and R. M. Parker

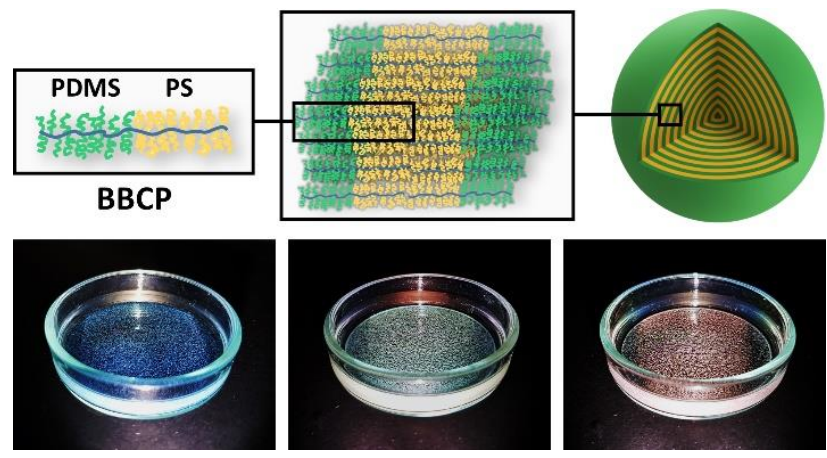

Bottlebrush block copolymers are selfassembled within micron-scale shrinking droplets. The produced microspheres form a well-ordered, concentric lamellar structure that intensely reflects iridescent color. The color is both tunable and responsive, making such microspheres ideal for use as a photonic pigment.

Keywords: Bottlebrush block copolymers, Photonic crystals, Confined self-assembly, Structural Colors, Microspheres 